\title{
PROGRESS ON DUAL HARMONIC ACCELERATION ON THE ISIS SYNCHROTRON
}

\author{
A. Seville, I. Gardner, J. Thomason, D. Adams, D. Bayley, C. Warsop, \\ STFC, Rutherford Appleton Laboratory, Chilton, Didcot, UK.
}

\section{Abstract}

The ISIS synchrotron at the Rutherford Appleton Laboratory in the UK is currently undergoing an RF upgrade. Four $\mathrm{h}=4$, or second harmonic ( $2 \mathrm{RF})$, cavities have been installed in addition to the existing six $\mathrm{h}=2$, fundamental frequency (1RF), cavities and should be capable of increasing the operating current from 200 to $300 \mu \mathrm{A}$. Two of the four cavities have been in operation for the last 2 user cycles of 2007 improving trapping losses and increasing operating currents beyond $200 \mu \mathrm{A}$. The remaining two cavities were commissioned in the spring of 2008. This paper reports on hardware commissioning, beam tests and beam simulation results.

\section{INTRODUCTION}

Over the last twenty years, acceleration of the ISIS synchrotron beam has been provided by six two-gap RF cavities. With this arrangement $\sim 2.75 \times 10^{13}$ protons can be held in the synchrotron throughout the $10 \mathrm{~ms}$ accelerating cycle from 70 to $800 \mathrm{MeV}$ during which the RF sweeps from 1.3 to $3.1 \mathrm{MHz}$. The maximum mean beam current which can be accelerated by the synchrotron is $\sim 220 \mu \mathrm{A}$, although for ease of active maintenance, beam loss limits the operational beam current to $\sim 180 \mu \mathrm{A}$. The addition of a second harmonic component $[1,2]$ to the RF waveform, as shown in figure 1 , should allow the acceleration of higher currents by extending the phase stable region and therefore increasing the bunching factor.

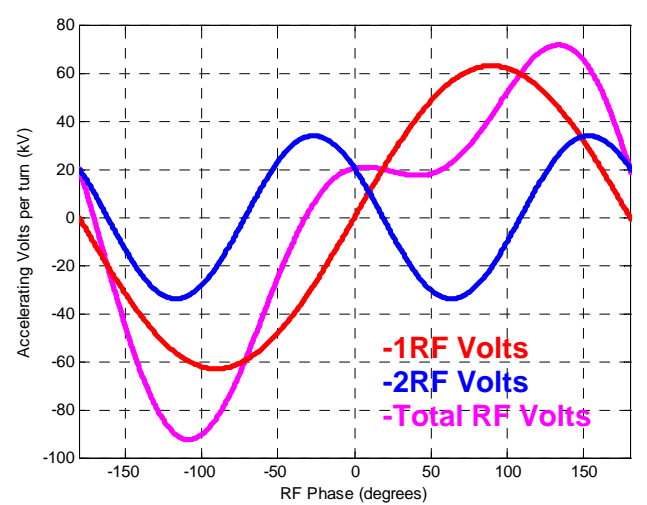

Figure 1: Addition of $1 \mathrm{RF}$ and $2 \mathrm{RF}$ components.

The longitudinal phase acceptance is increased due to the addition of the $2 \mathrm{RF}$ component, giving a higher trapping efficiency. Simulations indicate that up to $\sim 3.75 \times 10^{13}$ protons, or $\sim 6 \mu \mathrm{C}$ of protons, can be held and accelerated using this technique. In ISIS the $2 \mathrm{RF}$ component is provided by four $2 \mathrm{RF}$ cavities, installed in super-periods (SP) 4, 5, 6 and 8. The cavities are similar in design to the existing fundamental frequency cavities, but are approximately half the length. As with the 1RF cavities, the resonant frequency of the $2 \mathrm{RF}$ cavities has to sweep throughout the acceleration cycle (at twice the fundamental frequency, 2.6 to $6.2 \mathrm{MHz}$ ) to match the changing rotational frequency. This sweeping is effected, by loading the $2 \mathrm{RF}$ cavities with ferrite and then sweeping the ferrite bias current throughout the acceleration cycle to change the permeability of the ferrite and hence the inductive element of the equivalent L-C circuit. The hardware necessary for driving the new $2 \mathrm{RF}$ cavities is based on that used very successfully over the last twenty years for the fundamental cavities, but the electrical and electronic hardware has been updated where appropriate.

\section{COMMISSIONING THE 2RF SYSTEMS}

Since the last reported results [3], there has been much work done on the $2 \mathrm{RF}$ systems. Shortly after the successful operation of the $2 \mathrm{RF}$ systems giving dual harmonic accelerated beam of over $200 \mu \mathrm{A}$ in December 2006, further machine physics tests were hampered by a failure in the transformer of one of the $2 \mathrm{RF}$ anode power supply for the 2RF system in SP6, which rendered the SP6 2RF cavity unusable. Fortunately, the long shutdown from December 2006 to October 2007, scheduled for preparatory work on ISIS Target Station 2, gave an opportunity to replace the anode power supply with a spare unit. Other work on the $2 \mathrm{RF}$ systems was also carried out during the long shutdown. The low power RF (LPRF) systems were re-styled and housed in vertical CAMAC style crates, as shown in figure 2 , in order to reduce over-heating seen on the previous units. The bandwidths of the level control loops and tuning loops were adjusted to reduce the effect of loop oscillations on the RF signals. Further improvements were made to the four anode power supplies by replacing the banks of 88 small electrolytic capacitors by 2 oil-filled polypropylene film capacitors, as shown in figure 3 , to reduce the likelihood of the power supply tripping on current surges, and also to improve reliability of the power supplies.

The replacement power supply in 2RF system 6 was not fully commissioned by the end of the long shutdown, so during the ISIS operational cycle 2007/1 beam could only be accelerated with two second harmonic cavities (systems 4 and 5) in operation. However, even with only two systems providing the second harmonic component of the accelerating field, successful acceleration of beam pulses containing $2.93 \times 10^{13}$ protons was achieved during machine physics tests on November $1^{\text {st }}, 2007$. This beam intensity was achieved at low repetition rate $(1.6 \mathrm{~Hz})$, but pulses of the same intensity would give an equivalent $50 \mathrm{~Hz}$ beam current of $234 \mu \mathrm{A}$.

A04 Circular Accelerators 


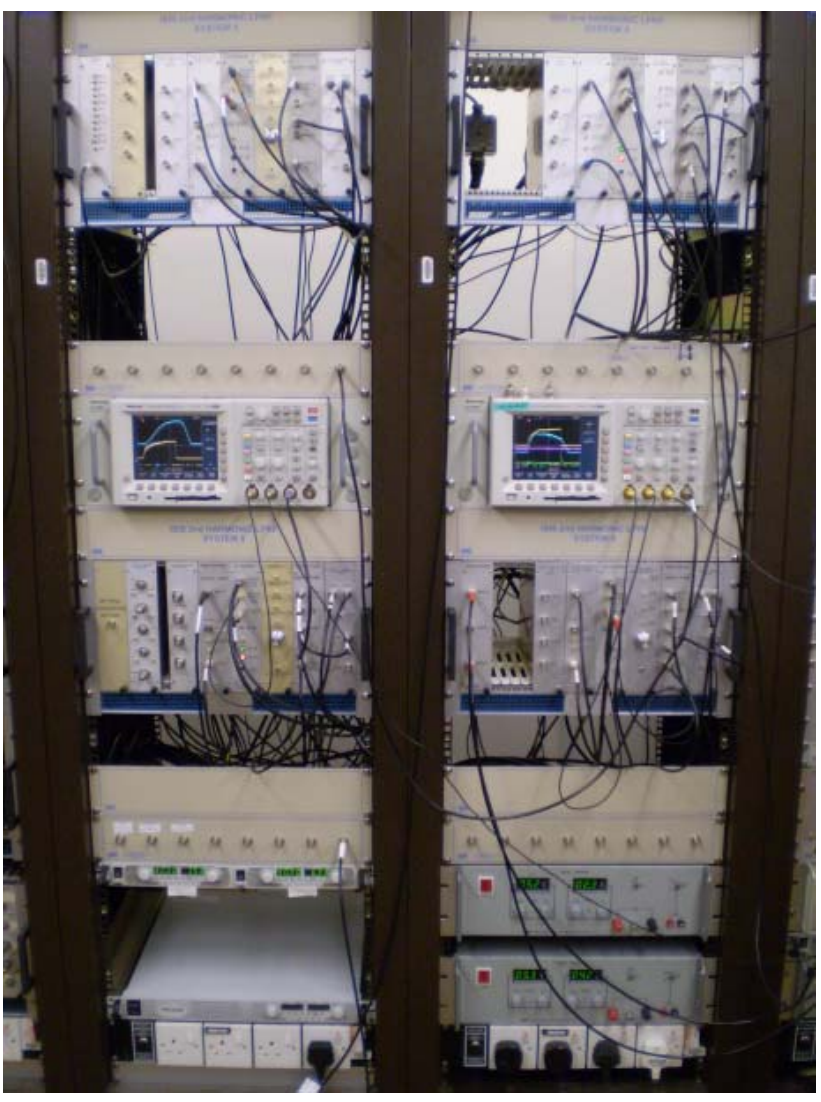

Figure 2: The replacement second harmonic low power $\mathrm{RF}$ units installed in the ISIS diagnostics room.

For these beam tests, the peak accelerating $2 \mathrm{RF}$ voltage per accelerating gap had been increased from $7.2 \mathrm{kV}$ to $9 \mathrm{kV}$. The total accelerating voltage per turn throughout the $10 \mathrm{~ms}$ acceleration period is shown in figure 4 .

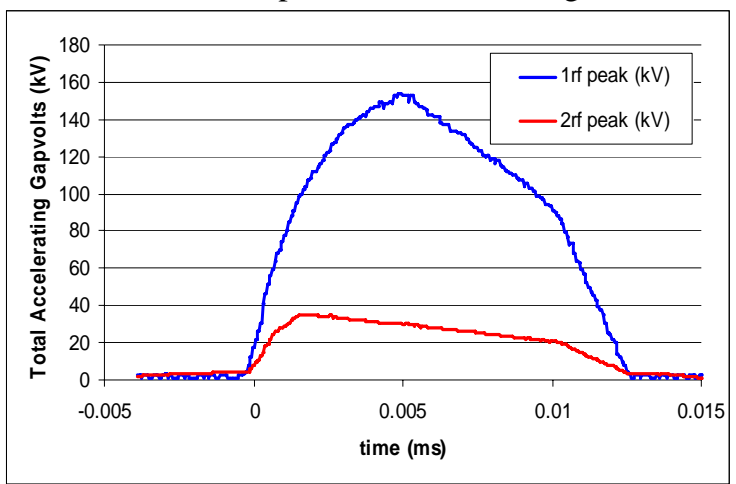

Figure 4: Total $1 \mathrm{RF}$ and $2 \mathrm{RF}$ accelerating voltages used to accelerate $234 \mu \mathrm{A}$ equivalent beam.

Further machine physics tests were planned and systems 6 and 8 we also made operational by increasing the accelerating voltage per gap from $7.2 \mathrm{kV}$ to $9 \mathrm{kV}$. However, just before tests with beam could be made, a repeat of the anode power supply transformer failure (this time in system 8) prevented operation. In the three subsequent operational cycles to date, when dual harmonic acceleration has been achieved, it has relied on only two second harmonic cavities.

04 Hadron Accelerators

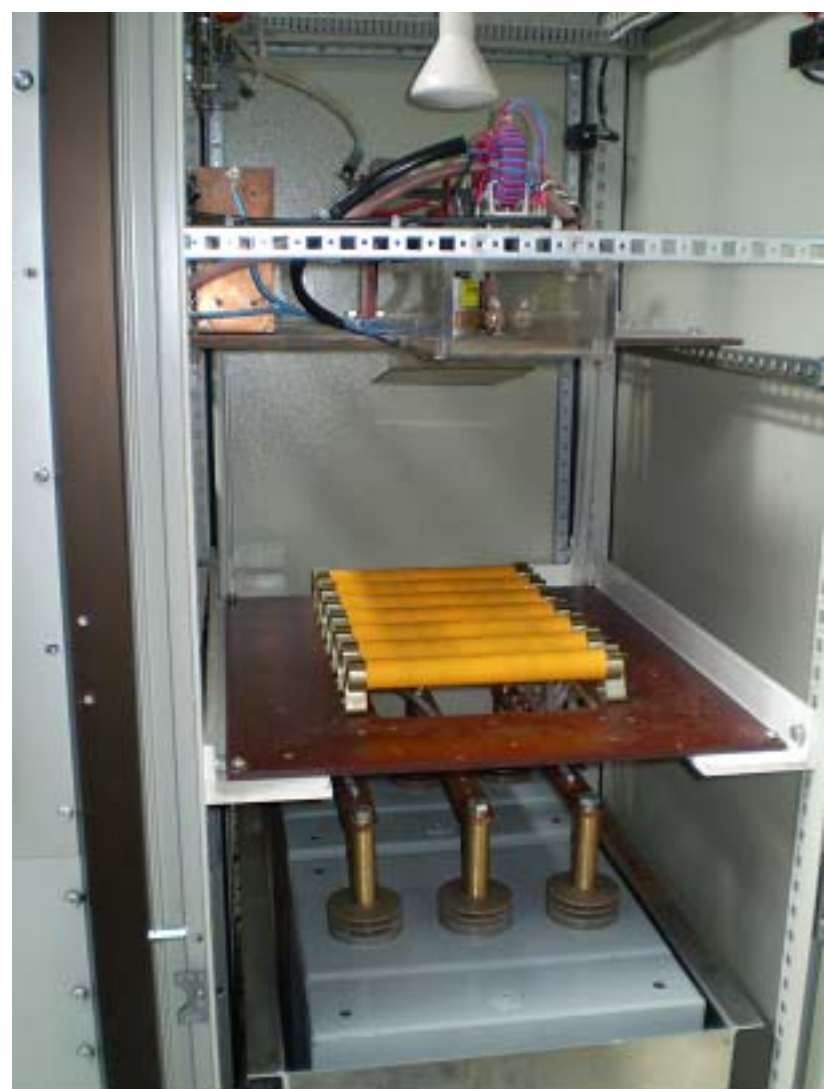

Figure 3: The replacement second harmonic anode power supply capacitors.

Nevertheless, dual harmonic acceleration enabled $50 \mathrm{~Hz}$ accelerated beam with an average current of over $200 \mu \mathrm{A}$ during the 24 hour period of February $29^{\text {th }}, 2008$. During the scheduled machine physics period from late March to early April and also in mid May 2008, further 2RF system tests were scheduled. During these periods, further commissioning of the beam compensation system was carried out. The feed-forward beam compensation system is a copy of that used on the $1 \mathrm{RF}$ systems [4] and is shown schematically in figure 5 . It comprises a beam pick-up electrode signal which is first filtered to generate a pure $2 \mathrm{RF}$ component. The signal is then amplified to an appropriate level and delayed sufficiently to cancel the beam loading of the proton bunch on the subsequent turn.

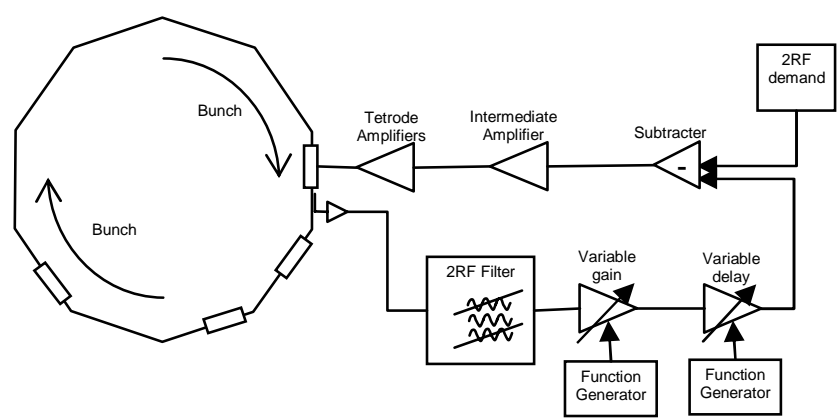

Figure 5: the feed-forward beam compensation system.

An attempt was made to replace the fixed band-pass filter with two switch-in filters in order to allow beam 
compensation to be applied during the early and late parts of the beam acceleration period. Tests with beam showed that whilst the system did indeed give cancellation of the beam induced voltages during the periods when each of the filters was switched in, the later part appeared unstable, possibly due to the $1 \mathrm{RF}$ component of the beam signal being passed by the high frequency filter. Further tests will be made and the possibility of using a tuneable digital filter is also being explored.

\section{OPERATIONAL DHRF RESULTS}

For the recent machine physics tests in May 2008, the failed transformer in $2 \mathrm{RF}$ system 8 had been replaced by a higher specification unit. However, an unrelated fault on the anode power supply for system 4 left only 3 operable $2 \mathrm{RF}$ cavities. Beam was accelerated at low repetition rate $(1.6 \mathrm{~Hz})$ at increasing intensity. Initial tests with $2 \mathrm{RF}$ systems 5 and 8 operating at $8.4 \mathrm{kV}$ peak voltage gave an accelerated beam of $2.67 \times 10^{13}$ protons, equivalent to a $50 \mathrm{~Hz}$ beam current of $212 \mu \mathrm{A}$. Increasing the peak voltages to $9 \mathrm{kV}$ per gap allowed an equivalent beam current of $220 \mu \mathrm{A}$ to be accelerated. The further addition of $2 \mathrm{RF}$ system 6 operating at $7.2 \mathrm{kV}$ peak gap voltage allowed an equivalent beam current of $228 \mu \mathrm{A}$ to be accelerated. Figure 6 shows the beam intensity monitor signal for these accelerated beam currents together with that for a single harmonic accelerated beam and the $234 \mu \mathrm{A}$ equivalent beam from November 2007. Figure 7 shows the corresponding beam loss monitor signals.

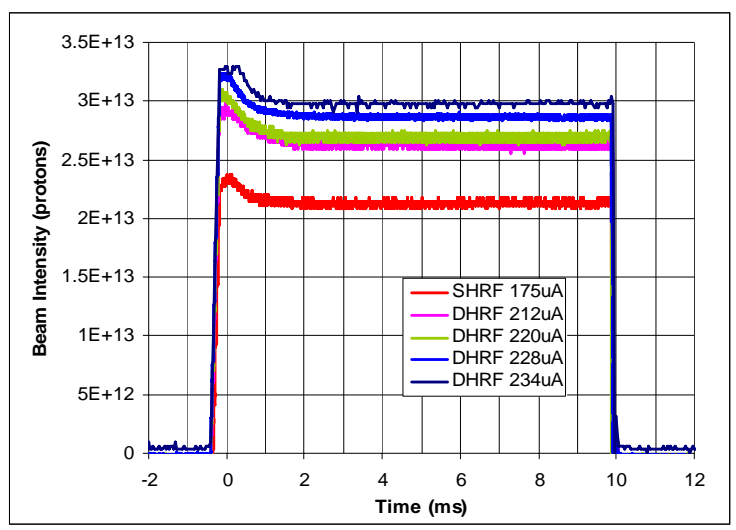

Figure 6: Accelerated beam intensity signals.

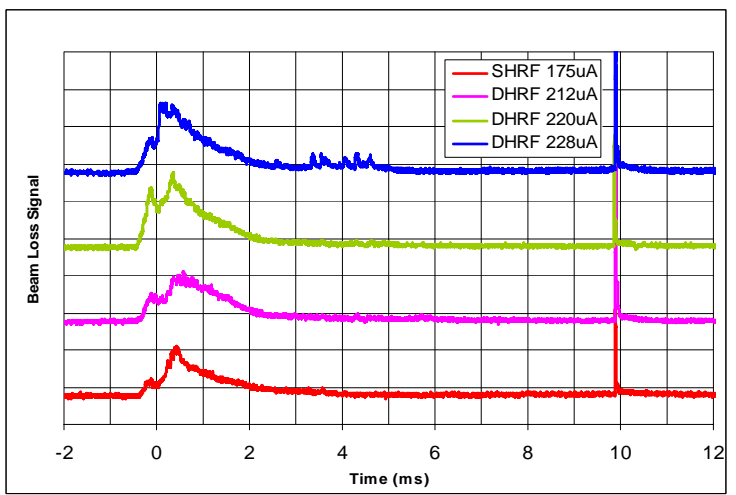

Figure 7: Accelerated beam intensity signals.
The beam loss monitor signals are shown here as a qualitative guide to the losses during ISIS acceleration. The initial peak corresponds to losses during injection and that just after $0 \mathrm{~ms}$ to trapping loss. The loss levels in each case shown are within acceptable limits for ISIS operation at $50 \mathrm{~Hz}$. The trace for $228 \mu \mathrm{A}$ shows some midcycle loss at 3-5ms, which was seen intermittently for the higher intensity beam. Mid to late cycle beam loss is to be avoided as the protons in ISIS have much higher energy later in the acceleration cycle, causing higher activation of the machine, and this loss prevented acceleration of beam with higher intensity than $228 \mu \mathrm{A}$ and the high losses were of a frequency that would prevent $50 \mathrm{~Hz}$ operation. It has subsequently been found that these mid cycle losses may be driven by a $\sim 10 \mathrm{kHz}$ oscillation in the beam phase loop and investigations are being made to remove this fault.

\section{CONCLUSIONS AND FURTHER WORK}

The four 2RF cavities and their services have now been installed in the ISIS synchrotron, and the $2 \mathrm{RF}$ systems commissioned. Reliable operation of the 2RF systems, particularly the anode power supplies, has proven a challenge. However, several hardware problems have been overcome and dual harmonic acceleration has been successfully achieved during the last 4 ISIS user cycles. All four 2RF systems are yet to be utilized together. However, beam has been successfully accelerated with different pairs of the $2 \mathrm{RF}$ cavities of sufficient beam intensities to provide the increased beam current required by the addition of the second target station at ISIS.

The application of beam compensation on the $2 \mathrm{RF}$ systems has so far proven successful but is only applied early in the acceleration cycle, due to the fixed bandwidth of the filter used. It is intended in the near future to use a tuneable filter to enable beam compensation throughout the acceleration period.

The "second generation" low power RF equipment developed for the 2RF system has been optimised and can now be duplicated and installed as replacements for ageing low power RF equipment incorporated at present in the fundamental RF systems. As part of this process, a replacement digital RF Master oscillator [5] has been designed and built and will be commissioned during the next few months.

\section{REFERENCES}

[1] M. Harold et al, "A Possible Upgrade for ISIS", PAC'97, Vancouver, 1997, p. 1021.

[2] C. Prior, "Studies of Dual Harmonic Acceleration in ISIS”, ICANS XII, RAL Report 94025, 1994, p. A11.

[3] A. Seville et al, "Progress on dual harmonic acceleration on the ISIS synchrotron", PAC'07, Albuquerque, 2007, p.1649.

[4] P. Barratt et al, "RF system and beam loading compensation on the ISIS synchrotron", EPAC 1990.

[5] C. Appelbee et al, "Digital master oscillator results for the ISIS synchrotron", PAC'07, Albuquerque, 2007, p.1649. 